\title{
History of Ureteral Stenting Negatively Affects the Outcomes of Extracorporeal Shockwave Lithotripsy. Results of a Matched-pair Analysis
}

\author{
Stavros Sfoungaristos, Ofer N. Gofrit, Dov Pode, Ezekiel H. Landau, \\ Vladimir Yutkin, Arie Latke, Mordechai Duvdevani \\ Department of Urology, Hadassah Hebrew University Medical Center, \\ Jerusalem, Israel \\ Received July 13, 2015; Accepted September 15, 2015.
}

Key words: Ureteral stent - Stone - Shockwave - Lithotripsy - Stone free

Abstract: To evaluate the impact of ureteral stenting history to the outcomes of extracorporeal shockwave lithotripsy, we retrospectively analysed patients who underwent shockwave lithotripsy with Dornier Gemini lithotripter between September 2010 and August 2012. Forty seven patients (group A) who had a double J stent which was removed just before the procedure were matchedpaired with another 47 patients (group B) who underwent shockwave lithotripsy having no stent history. The correlation between ureteral stenting history and stone-free rates was assessed. Stone-free rates were $68.1 \%$ and $87.2 \%$ for patients of group $A$ and $B$, respectively $(p=0.026)$. Postoperative complications were not different between groups. Multivariate analysis revealed that stone size $(p=0.007)$, stone location $(p=0.044)$ and history of ureteral stenting $(p=0.046)$ were independent predictors for stone clearance after shockwave lithotripsy. Ureteral stents adversely affect shockwave lithotripsy outcome, even if they are removed before the procedure. Stenting history should divert treatment plan towards intracorporeal lithotripsy.

Mailing Address: Stavros Sfoungaristos, MD., PhD., Department of Urology, Hadassah Hebrew University Medical Center, $5^{\text {th }}$ floor, Ein-Kerem, Jerusalem, Israel; Phone: +972 508946 695; e-mail: sfoungaristosst@gmail.com 


\section{Introduction}

Extracorporeal shockwave lithotripsy (SWL) represents an efficient and wellestablished treatment for patients with urolithiasis. The overall success rates have been reported to be more than $90 \%$ in some series (Ghoneim et al., 2010;Wang et al., 2011). The main advantage over ureteroscopic and percutaneous techniques is the non-invasive fashion combined with beneficial therapeutic results. On the other hand, a significant number of patients fail to reach a stone-free state after a single SWL session, and consequently more than one session may be required to obtain stone clearance, and in some cases, auxiliary interventions are needed. All of the above result in time consuming, increased cost and negatively affected quality of life.

Many studies have evaluated several clinical and laboratory parameters in an effort to reveal the factors affecting SWL outcomes (Kanao et al., 2006; Salman et al., 2007; Kacker et al., 2008; Sfoungaristos et al., 2012). Apart of stone size and location which represent the most evaluated and most significant parameters, ureteral stenting has been reported to affect SWL outcomes (Pryor and Jenkins, 1990; Musa, 2008; Shen et al., 2011), mainly due to the induction of local inflammation and edema, and reduction of ureteral peristalsis. However, insertion of a double J ureteral stent is mandatory in some cases, due to the presence of complications secondary to renal stones, such as acute renal failure, urosepsis and recurrent renal colics resistant to analgesic treatment. Furthermore, a number of urologists prefer putting stents in patients with large renal stones assuming that it may prevent post-SWL upper urinary tract obstruction due to steinstrasse formation.

In some cases, the urologist decides to remove the ureteral stent just before the procedure. These include patients with severe discomfort, cases in which a ureteral stone was pushed back to the kidney during stent placement, and that found to be smaller than previously assessed. There is a bibliographic gap regarding stone-free rates in patients in whom a ureteral stent had been inserted for any reason and was removed prior to SWL. In this manuscript, we evaluate the influence of history of ureteral stenting on the therapeutic efficacy of SWL, and we suggest parameters that may predict stone clearance in these patients.

\section{Patients and Methods}

After we obtained an Ethics Committee approval, we retrospectively reviewed a prospectively maintained database of patients who underwent an SWL in our institution between September 2010 and August 2012. Due to retrospective fashion of the study, there was no need to obtain informed consent. All procedures were made by a fourth generation electro-magnetic lithotripter (Gemini, Dornier MedTech, GmbH, Germany) and performed by the same operator under supervision of the same urologist. We identified 47 patients with a ureteral stent. The indication for stent insertion was the presence of recurrent and resistant renal 
colic. In all cases, the ureteral stent was removed before SWL (group A) due to patients' discomfort and severe lower urinary tract symptoms. In order to examine the potential role of ureteral stenting history on stone-free status after SWL, we matched these cases with another cohort of 47 patients (group B) based on stone location and size. Patients of group B underwent SWL without a stent and they did not have a stent before the procedure, as well.

Treatment protocol, regarding number and frequency of shockwaves, was based on company guidelines and was strictly followed in all cases. All operations were performed under sedation. In all patients, the stent was removed on the day of SWL. Standard follow-up was performed 4-6 weeks after SWL and was based on non contrast abdominal computer tomography scan for identification of residual fragments.

Several parameters, like patients' age and gender, stone size, location, side, number of shockwaves applied, and history of stenting were evaluated to assess their impact on SWL outcomes. The association of stenting history with postoperative complications, like pain, febrile urinary tract infections, renal failure, steinstrasse and subcapsular hematoma formation was analysed, as well.

Statistical analysis was performed by using SPSS version 17 (SPSS Inc., Chicago, IL, USA). The descriptive statistics are presented as the mean \pm standard deviation (SD) and interquartile range (IQR) for continuous variables and as the absolute and percent frequency for categorical variables. Mann-Whitney $U$ test was used to compare means between numerical groups and chi-square $\chi^{2}$ test for categorical variables. One-way ANOVA used to compare means when categorical variables had more than 2 results. A multivariate analysis was performed for the variables identified as statistically important in univariate analysis, using logistic regression. All tests were 2 -tailed with $\mathrm{p}<0.05$ considered statistically significant.

\section{Results}

The study cohort consisted of 62 male patients (66.0\%) and 32 females (34.0\%) with a mean age of 48.1 years. The mean stone size was $9.03 \mathrm{~mm}$. Forty eight stones $(51.1 \%)$ found on the left side and 46 of them (48.9\%) on the right side while 36 stones (38.3\%) were located in the kidney and 58 (61.7\%) in the ureter. Characteristics of patients of group A and B are presented in Table 1. No differences were found in the complications rates between groups (Table 2).

Stone-free rates were $68.1 \%$ for patients of group A and $87.2 \%$ for group B. This difference was statistical significant $(p=0.026)$. Univariate analysis of several parameters revealed that stone size $(p<0.001)$ and stone location in the ureter $(p=0.002)$ significantly associated with stone-free rates after SWL, as well (Table 3$)$. The above parameters entered a multivariate logistic regression analysis for the identification of independent predictors (Table 4). All 3 parameters can significantly predict stone clearance after SWL, with stone size $(p=0.007)$ found to be the most significant followed by stone location $(p=0.044)$ and stent history $(p=0.046)$. 
Table 1 - Characteristics of study cohort

\begin{tabular}{|c|c|c|c|}
\hline & Group A & Group B & $\mathrm{P}$ \\
\hline No. of patients, n (\%) & $47(50.0)$ & $47(50.0)$ & \\
\hline Gender, n (\%) & & & $0.663^{\mathrm{a}}$ \\
\hline male & $32(68.1)$ & $30(63.8)$ & \\
\hline female & 15 (31.9) & $17(36.2)$ & \\
\hline Age (years) & & & $0.636^{b}$ \\
\hline mean $\pm S D(I Q R)$ & $47.0 \pm 17.0(27)$ & $48.8 \pm 16.3(23)$ & \\
\hline Side, n (\%) & & & $1.000^{\mathrm{a}}$ \\
\hline right & 23 (48.9) & 23 (48.9) & \\
\hline left & $24(51.1)$ & $24(51.1)$ & \\
\hline Location, n (\%) & & & $1.000^{c}$ \\
\hline upper calyx & $4(50.0)$ & $4(50.0)$ & \\
\hline middle calyx & $2(50.0)$ & $2(50.0)$ & \\
\hline lower calyx & $6(50.0)$ & $6(50.0)$ & \\
\hline renal pelvis & $6(50.0)$ & $6(50.0)$ & \\
\hline proximal ureter & $13(50.0)$ & $13(50.0)$ & \\
\hline middle ureter & $2(50.0)$ & $2(50.0)$ & \\
\hline distal ureter & $14(50.0)$ & $14(50.0)$ & \\
\hline Size $(\mathrm{mm})$ & & & $0.894^{b}$ \\
\hline mean $\pm S D(I Q R)$ & $10.7 \pm 4.57(7)$ & $10.1 \pm 2.93$ & \\
\hline
\end{tabular}

Table 2 - Relation of ureteral stenting history and post-extracorporeal shockwave lithotripsy complications

\begin{tabular}{lccc}
\hline & Group A & Group B & $\mathrm{P}$ \\
\hline Overall complications, n (\%) & $5(10.6)$ & $3(6.38)$ & 0.460 \\
Fever, n (\%) & $0(0.00)$ & $0(0.00)$ & $\mathrm{n} / \mathrm{a}$ \\
Steinstrasse, n (\%) & $2(4.26)$ & $0(0.00)$ & 0.153 \\
Pain, n (\%) & $2(4.26)$ & $1(2.13)$ & 0.557 \\
Acute renal failure, n (\%) & $1(2.13)$ & $1(2.13)$ & 1.000 \\
Perinephric hematoma, n (\%) & $0(0.00)$ & $1(2.13)$ & 0.315 \\
\hline
\end{tabular}

All statistics were made by chi-square $\chi^{2}$ test; $\mathrm{n} / \mathrm{a}$ - not available

\section{Discussion}

SWL has been established as one of the first line therapeutical options for patients with renal or ureteral stones. Non invasive fashion and low morbidity are the main advantages of SWL against ureteroscopy and percutaneous nephrolithotripsy, while in selected patients it produces similar beneficial results, especially in the era of fourth generation lithotripters (Pemberton and Tolley, 2006; Nomikos et al., 2007). However, a non negligible number of patients will not benefit from SWL, even after multiple sessions. For this group of patients, delay in definitive therapy may add to 
Table 3 - Stone-free rates

\begin{tabular}{|c|c|c|c|}
\hline & Not stone-free & Stone-free & $P$ \\
\hline No. of patients, $n$ (\%) & $21(22.3)$ & 73 (77.7) & \\
\hline Gender, n (\%) & & & $0.136^{a}$ \\
\hline male & $11(52.4)$ & 51 (69.9) & \\
\hline female & $10(47.6)$ & $22(30.1)$ & \\
\hline Age (years) & & & $0.716^{\mathrm{b}}$ \\
\hline mean $\pm S D(I Q R)$ & $49.5 \pm 16.2(25)$ & $47.4 \pm 16.8(21)$ & \\
\hline Side, n (\%) & & & $0.720^{\mathrm{a}}$ \\
\hline right & $11(52.4)$ & 35 (47.9) & \\
\hline left & $10(47.6)$ & $38(52.1)$ & \\
\hline Location, n (\%) & & $22(30.1)$ & $0.002 * a$ \\
\hline kidney & $14(66.7)$ & $51(69.9)$ & \\
\hline ureter & 7 (33.3) & & \\
\hline Location within the ureter, $\mathrm{n}(\%)$ & & & $0.541^{\mathrm{a}}$ \\
\hline upper & $2(7.7)$ & $24(92.3)$ & \\
\hline mid & $1(25.0)$ & $3(75.0)$ & \\
\hline lower & $4(14.3)$ & $24(85.7)$ & \\
\hline No. of shockwaves & & & $0.113^{b}$ \\
\hline mean $\pm S D(I Q R)$ & $2295.2 \pm 347.1(350)$ & $2223.3 \pm 267.5(150)$ & \\
\hline Stenting history, n (\%) & & & 0.026 *a \\
\hline no & $6(28.6)$ & $41(56.2)$ & \\
\hline yes & $15(71.4)$ & $32(43.8)$ & \\
\hline
\end{tabular}

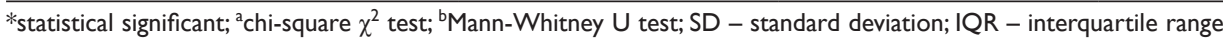

Table 4 - Multivariate analysis

\begin{tabular}{lccc}
\hline & $\mathrm{P}$ & Odds ratio & $95 \% \mathrm{Cl}$ \\
\hline Size & $0.007^{*}$ & 0.809 & $0.693-0.945$ \\
Location & $0.044^{*}$ & 0.301 & $0.094-0.970$ \\
History of stenting & $0.046^{*}$ & 0.302 & $0.093-0.981$ \\
\hline
\end{tabular}

*statistically significant; $\mathrm{Cl}$ - confidence interval

morbidity, in terms of recurrent pain and febrile infections of the upper urinary tract, cost and decreased quality of life. Furthermore, repeated SWL sessions may produce reversible histological alterations at ureteral epithelium and muscularis propria due to the induction of inflammation changes secondary to shockwaves (Portis et al., 2003) and consequently making secondary ureteroscopy technically more demanding. Determine these parameters which will characterize a certain group of patients who will mostly benefit from SWL would be beneficial for both patients and physicians. 
Several authors have attempted to identify predictive factors associated with success or failure of SWL. As we reported before, stone size and location are the most studied parameters and it is generally accepted that it may affect the outcomes of the procedure (Abdel-Khalek et al., 2003; Salman et al., 2007; Wang et al., 2011). In general, SWL is the first line option for stones $<10 \mathrm{~mm}$ (Salem, 2009). In contrast, the ideal management for stones greater than $10 \mathrm{~mm}$ remains to be defined, with both SWL and intracorporeal lithotripsy to represent an option. Abdel-Khalek et al. (2003) reported that stone transverse diameter $>10 \mathrm{~mm}$ is an adverse parameter for SWL success. Similarly, Delakas et al. (2003) found that stones larger than $10 \mathrm{~mm}$, located in the bony pelvis have decreased possibilities to be successfully managed by SWL.

Ureteral stents have been used as tools for relieving patients from recurrent renal colics and to prevent obstruction and consequently the danger of infected hydronephrosis and renal function deterioration. There is no generally accepted consensus for the benefits of stenting as a standard procedure prior to SWL. Ureteral stents are mainly avoided in cases with smaller stones while they are used for larger ones to prevent the consequences of steinstrasse and obstruction after treatment.

The potential effect of ureteral stenting on SWL success rates and postoperative morbidity represents a long standing issue of debate. The majority of published studies have reports that indwelling stents do not increase and even decrease stone-free rates. El-Assmy et al. (2006) reported a higher rate of stone clearance in the non-stented group of patient (91.4\%) compared to stented patients (84.9\%), although this difference did not reach statistical significant $(p=0.250)$. Ghoneim et al. (2010) observed similar results, as well. Nakada et al. (1995) evaluated the impact of ureteral stenting on expulsion of middle ureteral stones $<10 \mathrm{~mm}$ following SWL. They reported that there was no statistical difference in stone-free rate between the groups with the later to reach $82 \%$. In a contemporary prospective study, Sfoungaristos et al. (2012) assessed the need for pre-SWL routine stenting for ureteral stones with diameter $4-10 \mathrm{~mm}$. The authors reported $68.6 \%$ and $83.7 \%$ stone-free rates in stented and non-stented group $(p=0.026)$, respectively. Apart of this, stented patients underwent a greater number of SWL sessions $(p=0.019)$ while greater number of them underwent a definitive ureteroscopy due to SWL failure $(p=0.026)$. Ureteral stenting, stone size and location were the most significant predictors for stone clearance.

The histological background to support the theory that ureteral stenting may negatively affect the results of SWL is based on studies that have shown that double J stents react with ureteral epithelium and induce an inflammatory process. The latter results to edema formation, decrease in functional ureteral lumen diameter and consequently minimizes the likehood for stone passage (Auge et al., 2002). Stenting can also influence ureteral motility (Kinn and Lykkeskov-Andersen, 2002; Venkatesh et al., 2005; Natalin et al., 2009). Double J stents reduce peristaltic amplitude and frequency, as well as ureteral muscle tonicity. 
In the present study, we examined the effect of stenting history on SWL results. The question that prompted the study was if stents removal prior to SWL in these patients may alter SWL results. After an in depth search of the bibliography, we did not find studies which evaluate this question. Based on the results, it is revealed that success rates are significantly higher in these patients who were stent-free before and during SWL in contrast to patients who carried a ureteral stent before SWL and undergone the procedure without a stent. Furthermore, a stent history found to predict, in adjunct with stone size and location, stone-free status after extracorporeal lithotripsy. Additionally, the results of the present study support the hypothesis that stent removal prior to SWL may be beneficial for the patient.

The present study has some limitations that should be reported. The study is not a randomized controlled one and consequently parameters which may affect stone clearance, like computer tomography attenuation coefficient, stone composition, skin-to-stone distance, adjuvant medical expulsive therapy, were not studied. In addition, study population is not large and this may affect the study outcomes.

Follow-up time was not constant and evaluation for stone expulsion was not time-standardized. Although all procedures were performed by the same lithotripter and operator, stents were inserted by different urologists and this may constitute a limitation.

\section{Conclusion}

Reviewing the results of the present study, we can conclude that ureteral stents may adversely influence SWL results. In the present study, we showed that ureteral stents, even if they were removed just before the procedure, decrease $S W L$ stone-free rates. The routine use of ureteral stenting, irrespective of stone size and position, should not be considered. A history of ureteral stenting may be considered an indication for intracorporeal lithotripsy.

\section{References}

Abdel-Khalek, M., Sheir, K., Elsobky, E., Showkey, S., Kenawy, M. (2003) Prognostic factors for extracorporeal shock-wave lithotripsy of ureteric stones - a multivariate analysis study. Scand. J. Urol. Nephrol. 37, 413-418.

Auge, B. K., Ferraro, R. F., Madenjian, A. R., Preminger, G. M. (2002) Evaluation of a dissolvable ureteral drainage stent in a Swine model. J. Urol. 168, 808-812.

Delakas, D., Karyotis, I., Daskalopoulos, G., Lianos, E., Mavromanolakis, E. (2003) Independent predictors of failure of shockwave lithotripsy for ureteral stones employing a second-generation lithotripter. J. Endourol. 17, 201-205.

El-Assmy, A., El-Nahas, A. R., Sheir, K. Z. (2006) Is pre-shock wave lithotripsy stenting necessary for ureteral stones with moderate or severe hydronephrosis? J. Urol. 176, 2059-2062.

Ghoneim, I. A., El-Ghoneimy, M. N., El-Naggar, A. E., Hammoud, K. M., El-Gammal, M.Y., Morsi, A. A. (2010) Extracorporeal shock wave lithotripsy in impacted upper ureteral stones: a prospective randomized comparison between stented and non-stented techniques. Urology 75, 45-50.

Kacker, R., Zhao, L., Macejko, A., Thaxton, C. S., Stern, J., Liu, J. J., Nadler, R. B. (2008) Radiographic parameters 
on noncontrast computerized tomography predictive of shock wave lithotripsy success. J. Urol. 179, 1866-1871.

Kanao, K., Nakashima, J., Nakagawa, K., Asakura, H., Miyajima, A., Oya, M., Ohigashi, T., Murai, M. (2006) Preoperative nomograms for predicting stone-free rate after extracorporeal shock wave lithotripsy. J. Urol. 176, 1453-1456.

Kinn, A. C., Lykkeskov-Andersen, H. (2002) Impact on ureteral peristalsis in a stented ureter. An experimental study in the pig. Urol. Res. 30, 213-218.

Musa, A.A. (2008) Use of double-J stents prior to extracorporeal shock wave lithotripsy is not beneficial: results of a prospective randomized study. Int. Urol. Nephrol. 40, 19-22.

Nakada, S. Y., Pearle, M. S., Soble, J. J., Gardner, S. M., McClennan, B. L., Clayman, R.V. (1995) Extracorporeal shock-wave lithotripsy of middle ureteral stones: are ureteral stents necessary? Urology 46, 649-652.

Natalin, R. A., Hruby, G.W., Okhunov, Z., Singh, H., Phillips, C. K., Humphrey, P. A., Gupta, M., Landman, J. (2009) Pilot study evaluating ureteric physiological changes with a novel "ribbon stent" design using electromyographic and giant magnetoresistive sensors. BJU Int. 103, 1128-1131.

Nomikos, M. S., Sowter, S. J., Tolley, D. A. (2007) Outcomes using a fourth-generation lithotripter: a new benchmark for comparison? BJU Int. 100, 1356-1360.

Pemberton, R. J., Tolley, D. A. (2006) Comparison of a new-generation electroconductive spark lithotripter and the Dornier Compact Delta for ureteral calculi in a quaternary referral center. J. Endourol. 20, 732-736.

Portis, A. J., Yan, Y., Pattaras, J. G., Andreoni, C., Moore, R., Clayman, R. (2003) Matched pair analysis of shock wave lithotripsy effectiveness for comparison of lithotriptors. J. Urol. 169, 58-62.

Pryor, J. L., Jenkins, A. D. (1990) Use of double-pigtail stents in extracorporeal shock wave lithotripsy. J. Urol. 143, 475-478.

Salem, H. K. (2009) A prospective randomized study comparing shock wave lithotripsy and semirigid ureteroscopy for the management of proximal ureteral calculi. Urology 74, 1216-1221.

Salman, M., Al-Ansari, A. A., Talib, R. A., El-Malik, El-F., Al-Bozaom, I. A., Shokeir, A. A. (2007) Prediction of success of extracorporeal shock wave lithotripsy in the treatment of ureteric stones. Int. Urol. Nephrol. 39, 85-89.

Sfoungaristos, S., Polimeros, N., Kavouras, A., Perimenis, P. (2012) Stenting or not prior to extracorporeal shockwave lithotripsy for ureteral stones? Results of a prospective randomized study. Int. Urol. Nephrol. 44, 731-737.

Shen, P., Jiang, M., Yang, J., Li, X., Li, Y., Wei, W., Dai, Y., Zeng, H., Wang, J. (2011) Use of ureteral stent in extracorporeal shock wave lithotripsy for upper urinary calculi: a systematic review and meta-analysis. J. Urol. 186, 1328-1335.

Venkatesh, R., Landman, J., Minor, S. D., Lee, D. I., Rehman, J., Vanlangendonck, R., Ragab, M., Morrissey, K., Sundaram, C. P., Clayman, R.V. (2005) Impact of a double-pigtail stent on ureteral peristalsis in the porcine model: initial studies using a novel implantable magnetic sensor. J. Endourol. 19, 170-176.

Wang, M., Shi, Q., Wang, X., Yang, K., Yang, R. (2011) Prediction of outcome of extracorporeal shock wave lithotripsy in the management of ureteric calculi. Urol. Res. 39, 51-57. 